\title{
Tecnologia em aleitamento materno: revisão integrativa da literatura
}

\section{Technology in maternal breastfeeding: integrative review of the literature}

\author{
Erdnaxela Fernandes do Carmo Souza ${ }^{1}$ Elenice Valentim Carmona ${ }^{2}$ Maria Helena Baeno de Moraes Lopes ${ }^{3}$. \\ Antonieta Keiko Kakuda Shimo ${ }^{4}$
}

\begin{abstract}
RESUMO
Objetiva-se identificar as publicações da enfermagem brasileira em tecnologias no aleitamento materno. Trata-se de um estudo de revisão da literatura na área da saúde, com enfoque na revisão integrativa. Foram encontradas 18 referências, das quais 06 participaram da análise por atenderem aos critérios de seleção do estudo. Identificaramse 02 publicações que tratavam de Tecnologia Leve; 04 de Leve-Dura, e nenhuma de Tecnologia Dura. 0 Ceará foi o estado que apresentou o maior número de publicações com 05, seguido de São Paulo com 01. Os sujeitos da pesquisa foram: enfermeiros especialistas em aleitamento materno, puérperas e neonatos, mãe e pais cegos, e especialistas em conteúdo e em cordel. Conclui-se que a evolução da enfermagem no que diz respeito à produção científica em construção, validação, e adaptação, assim como, à influências de tecnologias educativas em saúde na promoção e apoio ao aleitamento materno, ainda encontra-se em número reduzido. Espera-se que os profissionais de saúde, em especial os enfermeiros, sintam-se estimulados a produção cientifica nessa temática, considerando resultados significativos para a prática profissional em aleitamento materno.
\end{abstract}

Palavras-chave: Aleitamento Materno; Tecnologia; Enfermagem.

\section{ABSTRACT}

The aim is to identify the brazilian nursing publications about technologies in breastfeeding. This is a review of the health literature with a focus on integrative review. We found 18 references, of which 06 participated in the analysis because they met the study selection criteria. We identified 02 publications dealing with Light Technology; 04 Light-Hard, and no Hard Tech. Ceará was the state that presented the highest number of publications with 05 , followed by São Paulo with 01. The subjects of the research were: nurses specialized in breastfeeding, recently given birth and neonates, mother and blind parents, and specialists in content and cordel. It concludes that the nursing evolution with regard to scientific production in construction, validation, and adaptation, as well as the influence of educational technologies on health in the promotion and support of breastfeeding are observed, although in a reduced number. It is expected that health professionals, especially nurses, will feel stimulated scientific production in this area, considering significant results for professional practice in breastfeeding.

Keywords: Breastfeeding; Technology; Nursing.

${ }^{1}$ Enfermeira Obstetra, Doutoranda do Programa de Pós-Graduação em Enfermagem da Faculdade de Enfermagem da Universidade Estadual de Campinas - UNICAMP. Campinas, Brasil. E-mail:enfxela@yahoo.com.br. Autora correspondente.

${ }^{2}$ Enfermeira Obstetra, Professora, Doutora da Faculdade de Enfermagem da Universidade Estadual de Campinas - UNICAMP. Campinas, Brasil. E-mail: carmona@unicamp.br 3 Enfermeira Obstetra, Professora Titular da Faculdade de Enfermagem da Universidade Estadual de Campinas - UNICAMP. Campinas, Brasil. E-mail: mhbaenaml@yahoo.com.br ${ }^{4}$ Enfermeira Obstetra, Professora Doutora da Faculdade de Enfermagem da Universidade Estadual de Campinas - UNICAMP. Campinas, Brasil. E-mail: akkshimo@gmail.com 


\section{INTRODUÇÃO}

As vantagens do aleitamento materno já são bastante reconhecidas na literatura, existindo um consenso mundial de que a sua prática exclusiva é a melhor maneira de proteger e nutrir adequadamente as crianças nos primeiros 6 meses de vida. Apesar dos esforços da Organização Mundial da Saúde (OMS) e o Fundo das Nações Unidas para a Infância (UNICEF) em proteger, promover e apoiar o Aleitamento Materno Exclusivo (AME), ainda está longe de ser uma realidade, segundo os dados de uma pesquisa sobre a prevalência do AME em menores de 6 meses foi de apenas $41 \%$, nas capitais brasileiras e no Distrito Federal (DF) ${ }^{1}$.

O apoio e promoção ao AME depende de esforços coletivos, intersetoriais, constituindo um grande desafio para os profissionais de saúde, principalmente para o enfermeiro, reservando a este profissional, um importante papel nos programas de educação em saúde, já desde o prénatal. Oportunidade em que ele pode atuar, desmistificando mitos e tabus, favorecendo adesão à amamentação, bem como promovendo momentos educativos no pós-parto imediato e no decorrer do puerpério, contribuindo com a manutenção do AME².

São várias as possíveis intervenções de enfermagem na promoção do AM, dentre as quais, podem ser classificadas como tecnologias aplicadas na assistência, que compreende a um conjunto de saberes e fazeres relacionado à produtos e materiais que definem terapêuticas e processos de trabalho e constituem-se em instrumentos para realizar ações na produção da saúde ${ }^{3}$.

As tecnologias envolvidas na assistência em saúde classificam-se em: Leve: quando referem-se às relações, acolhimento, gestão de serviços e produção de vínculo; Leve-Dura: relacionadas aos saberes bem estruturados, como o processo de enfermagem; Dura: material concreto, como máquinas, normas, estruturas organizacionais ${ }^{4}$.

A tecnologia mais apropriada será aquela que atenderá às necessidades de um grupo específico, com vistas à solucionar os problemas vivenciados ou já identificados em outro momento ${ }^{5}$.

De acordo com as perspectivas da utilização de tecnologias na assistência e promoção da saúde, fazse necessário incentivar a divulgação de tecnologias já construídas, que possam ser utilizadas pelos enfermeiros em sua assistência e estratégias de educação em saúde. Considerando estes aspectos, realizou-se uma revisão integrativa na literatura com objetivo de identificar as publicações da enfermagem brasileira em tecnologias no aleitamento materno.

\section{MÉTODO}

Trata-se de revisão integrativa da literatura, delimitando-se às seguintes etapas percorridas: (1) identificação do problema ou da temática (elaboração da pergunta norteadora, estabelecimento de descritores e dos critérios para inclusão/exclusão de artigos); (2) amostragem (seleção dos artigos); (3) categorização dos estudos; (4) definição das informações a serem extraídas dos trabalhos revisados; (5) análise e discussão a respeito das tecnologias utilizadas/desenvolvidas; (6) síntese do conhecimento evidenciado nos artigos analisados ou apresentação da revisão integrativa ${ }^{6}$.

Estabeleceu-se a seguinte pergunta norteadora: quais as publicações da enfermagem brasileira que utilizam a tecnologia no aleitamento materno? Os critérios de inclusão adotados pelo presente estudo foram: temática relacionada à tecnologia de enfermagem em aleitamento materno; disponibilidade eletrônica e gratuitamente na íntegra; artigo original; escritos em língua portuguesa. Foram excluídos os editoriais, cartas ao editor, estudos reflexivos, relatos de experiência, revisões, publicações duplicadas, teses e dissertações, bem como estudos que não abordassem a temática relevante ao objetivo da revisão.

As bases de dados utilizadas foram: Literatura LatinoAmericana e do Caribe em Ciências da Saúde (LILACS), Bases de Dados de Enfermagem (BDENF), além de bibliotecas virtuais como a Scientific Eletronic Library Online (SciELO), com abrangência nos últimos dez anos.

Utilizou-se a terminologia em saúde consultada nos Descritores em Ciências da Saúde (DeCS), pelos quais identificaram-se os respectivos descritores: aleitamento materno (breastfeeding); tecnologia (technology); enfermagem (nursing). As bases de dados utilizadas permitiram que realizasse uma busca avançada com três descritores ao mesmo tempo.

A busca ocorreu no mês de agosto de 2016, resultando em 18 referências levantadas nas referidas bases de dados e na biblioteca virtual SciELO. Destas, 12 foram excluídas por não corresponderem aos critérios de inclusão $(03$ relatos de experiência, 02 artigos de revisão, 05 repetiamse em outras bases de dados, e 02 teses).

Para a seleção das publicações, inicialmente, leu-se cada título e resumo para confirmar se eles contemplavam a pergunta norteadora desta investigação e se atenderiam aos critérios de inclusão e exclusão estabelecidos.

As 06 referências elegíveis de acordo com os critérios de inclusão, constituíram a amostra do estudo. Foram examinadas mediante um formulário adaptado de Ursi ${ }^{7}$. Este possibilitou a coleta de dados quanto aos seguintes aspectos: identificação do estudo, título do artigo, autores, ano de publicação, localidade do estudo, tipo de revista científica, características metodológicas, tecnologia utilizada/desenvolvida e os sujeitos da pesquisa.

Os artigos foram organizados por ano de publicação (iniciado pelos mais recentes) e fixados aos formulários correspondentes. A análise dos dados exigiu leitura e releitura dos artigos, realizaram-se operações estatísticas simples de distribuição de frequência absoluta e relativa. A seguir apresentam-se os principais dados. 


\section{RESULTADOS}

Por meio da análise dos 06 artigos selecionados, verificou-se que 03 estudos foram encontrados na base de dados LILACS, 02 na SciELO e 01 na BDENF. Observouse que as publicações ocorrem a partir do ano de 2008 , prevalecendo publicações no ano de 2013, realizados por enfermeiros brasileiros, como visualiza-se na figura 1.

Já em relação aos estados brasileiros em que foram realizadas as produções científicas, prevaleceu o estado do Ceará com 05 publicações e 01 em São Paulo. Quanto aos tipos de estudos foram: 02 quantitativos, 03 metodológicos (pré-experimental, validação, psicométrica) e 01 descritivo retrospectivo.

Verificou-se que alguns artigos trazem publicações da mesma tecnologia, assim foram identificados: Tecnologias Educativas em Saúde utilizando literatura de cordel sobre amamentação, bem como a Tecnologia Assistida cordel cantarolado, observa-se a evolução de construção, avaliação e adaptação cultural dessa tecnologia. Outra Tecnologia Educativa em Saúde: álbum seriado na promoção do aleitamento materno, assim como a Tecnologia Ilustrada: manual educativo para a promoção do aleitamento materno, e de promoção do aleitamento materno na primeira hora de vida. Já em relação aos sujeitos da pesquisa, encontrouse: enfermeiros especialistas em amamentação, puérperas e neonatos, mãe e pais cegos, e especialistas em conteúdo e em cordel, demonstrado na figura 2.

De acordo com as tecnologias desenvolvidas nas publicações deste estudo, foi possível classifica-las conforme Merly ${ }^{4}$, em: Tecnologia Leve: Tecnologia Assistiva: Cordel Cantarolado ${ }^{5}$; Promoção do Aleitamento Materno na primeira hora de vida ${ }^{6}$. Tecnologia Leve-Dura: Tecnologia Educativa em Saúde: Álbum Seriado "Eu posso amamentar o meu filho" ${ }^{2}$; Tecnologia Ilustrada: Manual
Educativo para a Promoção do Aleitamento Materno3; Tecnologias Educativas em Saúde: Literatura de Cordel sobre Amamentação ${ }^{1-4}$, como observa-se na figura 2.

\section{DISCUSSÃO}

A enfermagem vem utilizando tecnologias em saúde na promoção do aleitamento materno, e conhecendo as influências de tecnologias também como estratégias em educação. Sendo assim, foram identificados 02 tipos de tecnologias nos estudos analisados (leve e leve-dura) de acordo com a classificação das tecnologias envolvidas na assistência em saúde ${ }^{4}$, não sendo encontrado nesse estudo tecnologia dura, conforme a seguir:

\section{Tecnologia leve em aleitamento materno}

Nos estudos classificados em tecnologia leve, percebe-se a importância de uma inovação nos meios de comunicação, de forma que as orientações possam ser cantaroladas em cordel sobre a amamentação $0^{5}$, utilizada como instrumento de comunicação e educação em saúde a deficientes visuais. Espera-se que esta prática seja difundida entre os profissionais da saúde, de modo que a tecnologia assistiva seja capaz de ampliar as habilidades funcionais e a autonomia de pessoas com deficiência, além de viabilizar a inclusão social ${ }^{8}$.

0 aleitamento materno deve ser iniciado nas primeiras duas horas de vida, ainda na sala de parto, se a mãe e o recém-nascido estiverem em boas condições de saúde, favorecendo o contato pele a pele. Entretanto, a amamentação na primeira hora de vida pode sofrer influências negativas em relação à algumas práticas hospitalares e uso de tecnologias modernas como citada em um estudo ${ }^{6}$ : condutas intervencionistas (uso

Figura 1: Distribuição das referências incluídas na revisão integrativa (enumeradas) de acordo com as bases de dados, ano de publicação, estados brasileiros, autores, periódicos, área de atuação e tipo de estudo. Campinas-SP, 2016.

\begin{tabular}{|c|c|c|c|c|c|c|}
\hline Bases de dados & Ano & Estado (Brasil) & Autor & Periódico & Área de Atuação & Tipo de estudo \\
\hline $\begin{array}{c}(1) \\
\text { SciELO }\end{array}$ & 2014 & $\begin{array}{c}\text { Ceará } \\
\text { (Doutorado sanduiche em } \\
\text { Portugal). }\end{array}$ & Oliveira et al. & $\begin{array}{c}\text { Texto Contexto } \\
\text { Enferm. }\end{array}$ & Enfermagem & Quantitativo \\
\hline $\begin{array}{c}(2) \\
\text { LILACS }\end{array}$ & 2013 & Ceará & Dodt et al. & $\begin{array}{c}\text { Texto Contexto } \\
\text { Enferm. }\end{array}$ & Enfermagem & $\begin{array}{c}\text { Quantitativo } \\
\text { (Pré-experimental) }\end{array}$ \\
\hline $\begin{array}{c}(3) \\
\text { BDENF }\end{array}$ & 2013 & Ceará & Costa et al. & Rev. Rene & Enfermagem & $\begin{array}{c}\text { Metodológico } \\
\text { (Validação) }\end{array}$ \\
\hline $\begin{array}{c}(4) \\
\text { LILACS }\end{array}$ & 2013 & Ceará & Oliveira et al. & $\begin{array}{c}\text { Rev. Esc. Enferm } \\
\text { USP }\end{array}$ & Enfermagem & $\begin{array}{c}\text { Metodológico } \\
\text { (Psicometria) }\end{array}$ \\
\hline $\begin{array}{c}(5) \\
\text { SciELO }\end{array}$ & 2009 & Ceará & Oliveira et al. & $\begin{array}{c}\text { Rev. Bras. Enferm. } \\
\text { REBEn }\end{array}$ & Enfermagem & Metodológico \\
\hline $\begin{array}{c}(6) \\
\text { LILACS }\end{array}$ & 2008 & São Paulo & Pillegi & Einstein SP & Enfermagem & Descritivo \\
\hline
\end{tabular}

Fonte: dados da pesquisa. 
Figura 2: Distribuição das referências incluídas na revisão integrativa (numeradas), de acordo com o título do artigo, tipo de tecnologia aplicada e sujeitos da pesquisa. Campinas-SP, 2016.

\begin{tabular}{|c|c|c|}
\hline Título do artigo & Tipo de Tecnologia & Sujeitos da Pesquisa \\
\hline $\begin{array}{l}\text { (1) Adaptação cultural de tecnologia } \\
\text { educativa em saúde literatura de cordel } \\
\text { com enfoque na amamentação. }\end{array}$ & $\begin{array}{c}\text { Tecnologia Leve Dura } \\
\text { Tecnologia Educativa em Saúde } \\
\text { (Literatura de cordel sobre amamentação) }\end{array}$ & $\begin{array}{c}\text { Enfermeiras } \\
\text { (Juízas - Mestres e Doutoras) }\end{array}$ \\
\hline $\begin{array}{l}\text { (2) Influência de estratégia de educação } \\
\text { em saúde mediada por álbum seriado } \\
\text { sobre a autoeficácia materna para } \\
\text { amamentar. }\end{array}$ & $\begin{array}{c}\text { Tecnologia Leve Dura Tecnologia } \\
\text { Educativa em Saúde } \\
\text { (Álbum seriado “Eu posso amamentar o } \\
\text { meu filho") }\end{array}$ & Puérperas/Neonatos \\
\hline $\begin{array}{l}\text { (3) Construção e validação de manual } \\
\text { educativo para a promoção do } \\
\text { aleitamento materno. }\end{array}$ & $\begin{array}{l}\frac{\text { Tecnologia Leve Dura }}{\text { Tecnologia Ilustrada }} \\
\text { (Manual educativo para a promoção do } \\
\text { aleitamento materno) }\end{array}$ & $\begin{array}{c}\text { Profissionais de saúde (especialista em } \\
\text { aleitamento materno) } \\
\text { Puérperas }\end{array}$ \\
\hline $\begin{array}{l}\text { (4) Avaliação de tecnologia educativa na } \\
\text { modalidade literatura de cordel sobre } \\
\text { amamentação. }\end{array}$ & $\begin{array}{c}\frac{\text { Tecnologia Leve Dura }}{\text { Tecnologia Educativa }} \\
\text { (Literatura de cordel sobre amamentação) }\end{array}$ & $\begin{array}{l}\text { Juízes especialistas em conteúdo } \\
\text { (doutores) } \\
\text { Juízes cordialistas } \\
\text { (Pós-graduação/especialista em cordéis) }\end{array}$ \\
\hline $\begin{array}{l}\text { (5) Construção de uma tecnologia } \\
\text { assistiva para validação entre cegos: } \\
\text { enfoque na amamentação. }\end{array}$ & $\begin{array}{c}\text { Tecnologia Leve } \\
\text { Tecnologia Assistiva (Cordel cantarolado) }\end{array}$ & $\begin{array}{l}\text { Mães e pais } \\
\text { Cegos }\end{array}$ \\
\hline $\begin{array}{l}\text { (6) A amamentação na primeira hora de } \\
\text { vida e a tecnologia moderna: prevalência } \\
\text { e fatores limitantes. }\end{array}$ & $\begin{array}{c}\text { Tecnologia Leve } \\
\text { Promoção do Aleitamento Materno } \\
\text { na } 1^{\text {a }} \text { hora de vida }\end{array}$ & Mães de recém-nascidos sadios \\
\hline
\end{tabular}

Fonte: dados da pesquisa.

excessivo de exames de ultrassonografia, anestesias, analgesias, ocitocinas, monitorização fetal continua, partos cesárea), além do número elevado de partos diários, e a indisponibilidade de espaços adequados para a promoção do aleitamento materno no pós-parto imediato. Contudo, reforça-se a necessidade de intervenções de enfermagem, no apoio e acolhimento as mães e neonatos, utilizandose de tecnologia leve para que de fato seja possível estabelecer vínculos, e minimizar fatores limitantes a promoção do aleitamento materno.

\section{Tecnologia leve-dura em aleitamento materno}

Ficou evidente a importância da construção e validação de uma tecnologia ilustrada na elaboração de um manual educativo ${ }^{3}$, realizado a partir de saberes bem estruturados, como o processo de enfermagem em saúde, sendo capaz de responder as principais dúvidas que permeiam o cotidiano das mães para a promoção do aleitamento materno.

Comumente ainda são várias as causas que influenciam negativamente 0 aleitamento materno, conhecidas na literatura, bem como ao déficit de conhecimento materno sobre a amamentação ${ }^{9}$. Portanto, a elaboração e implementação de tecnologias que busquem promover o conhecimento em aleitamento materno pode contribuir com a melhoria dos índices desta prática e, consequentemente contribuir para a redução da morbimortalidade infantil ${ }^{3}$.

A utilização diária de tecnologias leve-dura pode facilitar tarefas antes consideradas impossíveis, assim como a construção de conhecimentos à serem socializados ${ }^{10}$. Tecnologia educativa sobre a amamentação no formato de literatura de cordel, por exemplo, vem sendo avaliada 4 por suas inúmeras vantagens, dentre elas, ser capaz de atrair o público pela rima, aguçar o interesse e a capacidade de construção de um instrumento de educação em saúde.

Os cordéis podem incentivar a prática da amamentação, a qual é considerada saudável e deve ser estimulada. 0 cordel em forma de versos como citado em um estudo ${ }^{4}$ construído por meio de saberes bem estruturados de especialistas, considerado um gênero literário, podendo ser escritos em versos ou cantarolado, e deve estar ao alcance de todos, dos mais cultos aos menos.

Em outro estudo ${ }^{1}$, foi realizada uma adaptação linguística cultural de uma produção científica brasileira em tecnologia educativa em saúde, a literatura de cordel sobre amamentação. Percebeu-se com isso, uma importante estratégia de promoção da saúde, mas que para utilizá-la, faz-se necessário a presença de profissionais de saúde para qualquer esclarecimento, bem como, conhecer o perfil das pessoas que irão utilizá-la

Sabe-se que o aleitamento materno possui importantes fatores de proteção, imunização, sendo capaz de suprir todas as necessidades biológicas e psicossociais para o bom crescimento e desenvolvimento da criança ${ }^{11-14}$.Para o sucesso desta prática, faz-se necessário além do conhecimento das mães, a autoeficácia em praticar o aleitamento materno. De acordo com um estudo² a autoeficácia pode ser influenciada por intervenções, mediante características especificas da 
população, podendo resultar em diferenças significativas na autoeficácia em amamentar.

Estratégias educativas pautadas na autoeficácia são relevantes na assistência de enfermagem, considerando o contexto em que as mulheres estão inseridas, bem como se recomenda a utilização de um álbum seriado, com objetivo de minimizar possíveis dificuldades no processo de amamentação, por meio de orientações bem estruturadas, possibilitando a utilização de materiais concretos de visualização, de modo que as puérperas saiam da maternidade mais seguras².

\section{CONCLUSÃO}

Observa-se a evolução da enfermagem no que diz respeito à produção científica em construção, validação, adaptação e influências de tecnologias educativas na promoção e apoio ao aleitamento materno, ainda que em número reduzido. Foram classificadas neste estudo como tecnologias: leve e leve-dura em aleitamento materno de acordo com a classificação de tecnologias envolvidas na assistência em saúde.

$\mathrm{Na}$ enfermagem, as tecnologias de educação em saúde são capazes de gerar impactos significativos no aleitamento materno. No entanto, o conceito de tecnologia pode estar sendo interpretado de forma equivocada, sendo atribuído apenas como máquina ou produto, apesar de já se observar mudanças nesse sentido não foi identificada tecnologia dura nesse estudo, ou até mesmo pode se encontrar dificuldades de como classifica-las na assistência, devendo assim justificar o número reduzido de publicações da enfermagem brasileira sobre tecnologias em aleitamento materno.

Espera-se que os profissionais de saúde, em especial os enfermeiros, sintam-se estimulados à produção científica nesta temática, considerando os resultados significativos identificados neste estudo para a prática profissional em aleitamento materno.

\section{REFERÊNCIAS}

1. Brasil. Ministério da Saúde. II Pesquisa de Prevalência de Aleitamento Materno nas Capitais Brasileiras e Distrito Federal. Brasília: Editora do Ministério da Saúde [Internet] 2009 [acesso em: 10 jun 2016]. Disponível em: http://portal. saúde. gov.br/portal/ arquivos/pdf/pesquisa_pdf.pdf.

2. BRASIL. Ministério da Saúde. Como ajudar as mães a amamentar. Brasília [Internet]. 2001 [acesso em: 10 jun 2016]. Disponível em: http://www.sbp.com.br/src/ uploads/2012/12/cd03_13.pdf

3. Nietsche EA. Tecnologia emancipatória: possibilidade para a práxis de enfermagem. Ijuí: Ed. UNIJUí; 2000.

4. Merhy EE. Em busca de ferramentas analisadoras das Tecnologias em Saúde: a informação e o dia a dia de um serviço, interrogando e gerindo trabalho em saúde. In: Merhy EE, Onoko, R, organizadores. Agir em Saúde: um desafio para o público. 2a ed. São Paulo (SP): Hucitec; 2002. p. 113 - 150.
5. Nietsche EA. Tecnologia emancipatória: possibilidade ou impossibilidade para a práxis de enfermagem. Florianópolis: UNIJUÍ; 2000.

6. Whittemore $\mathrm{R}$, Knafl $\mathrm{K}$. The integrative review: updated methodology. J. Adv. Nurs. 2005;52(5):54653.

7. Ursi ES. Prevenção de lesões de pele no perioperatório: revisão integrativa da literatura [dissertação]. Ribeirão Preto: Departamento de Enfermagem Geral e Especializada, Universidade de São Paulo, 2005.

8. Brasil. Decreto $n^{\circ}$. 6.215 , de 26 de setembro de 2007 [Internet]. 2007 [acesso em: 05 mar 2016]. Disponível: http://www.planalto.gov.br/ccivil_03/_Ato20112014/2011/ Decreto/D7612. htm\#art15.

9. Chaves RG, Lamounier JÁ, César CC. Fatores associados com a duração do aleitamento materno.J Pediatr. 2007;83(3):241-6.

10. Caetano JÁ, Pagliuca LMF. Cartilha sobre autoexame ocular para portadores de HIV/AIDS como tecnologia emancipatória: relato de experiência. Ver. Eletr Enferm. 2006; 8(2): 241-49.

11. World Health Organization: The World Health Organizations Infant-feeding recommendation. Bull World Health Organ. 1995; 73:165-74.

12. American Academy of Pediatrics. Breastfeeding and the use of human milk: Work group on breastfeeding. Pediatrics. 1997; 100: 1035-9.

13. Brasil. Ministério da Saúde, Saúde da Criança: Nutrição Infantil. Aleitamento Materno e Alimentação Complementar. Caderno de Atenção Básica n² 23. Brasília, 2009; 112p.

14. Victora CG. Infection and disease: The impact of early weaning. Food Nutr Bull. 1996; 17: 390-6. 